\title{
Some fuzzy common fixed point theorems using common limit in the range property with an application
}

\author{
Ved Prakash BhardwaJ, Kamal WadhWA
}

\begin{abstract}
In the present paper, we prove some common fixed point theorems for mappings satisfying common limit in the range property in $M$-fuzzy metric space. Further, we prove fixed point theorem for $\phi$-contractive conditions in aforesaid spaces with the illustration of an example. As an application of our result, we study the existence and uniqueness of the solution of integral equation (Volterra integral equations of the second kind) with instances.
\end{abstract}

\section{INTRODUCTION}

There are many generalizations of metric spaces. One of the generalization is introduced by Gahler [6] called 2-metric space. Dhage in 1992 introduced $D$-metric space [5], in his Ph.D. thesis and claimed that $D$-metric give a generalization of ordinary metric function and moved on to present some fixed point results. Afterwards, his works have been the foundation for several papers by Dhage himself and other authors. However, in 2004 Mustafa and Sims [13] demonstrated that most of the claims regarding the fundamental topological properties of $D$-metric spaces are incorrect, for example, a $D$ metric need not be a continuous function of its variables. Unfortunately, approximately all theorems in $D$-metric spaces are not valid see [15]. A modification in the definition of $D$-metric space was introduced by Sedghi et al. [21] as $D^{*}$-metric space. In view of [23], Sedghi and Shobe [22] given a concept of $M$-fuzzy metric space which is a fuzzy version of $D^{*}$-metric space and proved common fixed point theorems. Some recent results in M-fuzzy metric spaces are [14, 23].

On the other hand, Sintunavarat and Kumam [24] gave the notion of common limit in the range property as a generalization of property E.A. [1], which relaxes the condition of closedness of the underlying subspace.

2010 Mathematics Subject Classification. Primary: 54H25; Secondary: 47H10.

Key words and phrases. M-fuzzy metric spaces, Common limit in the range property ((CLR) property), Weakly compatible mappings.

Full paper. Received 31 January 2020, revised 9 March 2020, accepted 23 May 2020, available online 12 June 2020 . 
Besides these many scientific and engineering problems can be described by integral equations. Integral equations can also be formed by many mathematical physics models such as scattering in quantum mechanics, conformal mapping, wave water and diffraction problems. There are many areas that can be described by integral equations for instance propagation of stocked fish in a new lake, the heat radiation, biological species living together, Volterra's population growth model etc. Most of the initial and boundary value problems can be changed into Volterra or Fredholm integral equations. A class of mathematicians are working in this area and trying to obtain solutions of the integral equations using their results. Recently, Mishra et al. [12] introduce the notion of a modified $\alpha$ - $\phi$-fuzzy contractive mapping and prove some results in fuzzy metric spaces for such kind of mappings. As an application, they study the existence and uniqueness of the solution to integral equation. Some applications of fixed point theorems in metric or fuzzy metric theory can be seen in $[2,3,4,7,9,11,12,16,17,18,25]$.

In this paper, we establish some common fixed point theorems for mappings satisfying common limit in the range property under different contractive conditions in $M$-fuzzy metric space with the illustration of some examples. As an application of our result, we study the existence and uniqueness of the solution of integral equation (Volterra integral equations of the second kind) with examples.

\section{Preliminaries}

In this section, we have recalled some definitions and useful results which have already been in the literature.

Definition 1 ([19]). A binary operation $*:[0,1] \times[0,1] \rightarrow[0,1]$ is continuous $t$-norm if $*$ satisfying the following conditions:

(i) $*$ is commutative and associative;

(ii) $*$ is continuous;

(iii) $a * 1=a$, for all $a \in[0,1]$;

(iv) $a * b \leq c * d$, whenever $a \leq c$ and $b \leq d, \forall a, b, c, d \in[0,1]$.

Examples of $t$-norm are $a * b=a b$ and $a * b=\min (a, b)$.

Definition $2([8])$. A 3 -tuple $\left(X, M_{g}, *\right)$ is called a fuzzy metric space if $X$ is an arbitrary set, $*$ is a continuous $t$-norm, and $M_{g}$ is a fuzzy set in $X^{2} \times(0, \infty)$ satisfying the following conditions $\forall x, y \in X$ and $t>0$ :

(1) $M_{g}(x, y, t)>0$;

(2) $M_{g}(x, y, t)=1, t>0$ if and only if $x=y$;

(3) $M_{g}(x, y, t)=M_{g}(y, x, t)$;

(4) $M_{g}(x, y, t)^{*} M_{g}(y, z, s) \leq M_{g}(x, z, t+s)$;

(5) $M_{g}(x, y, \cdot):(0, \infty) \rightarrow[0,1]$ is continuous. 
Example 1 ([20]). Let $X$ be an arbitrary non-empty set and $\eta$ be an increasing and a continuous function of $R^{+}$into $(0,1)$ such that $\lim _{t \rightarrow \infty} \eta(t)=1$. Two typical examples of these functions are

$$
\eta(x)=\frac{x}{x+1}, \quad \text { and } \quad \eta(x)=1-e^{-x} .
$$

Denote $a * b=a b$, for all $a, b \in[0,1]$. For each $t \in(0, \infty)$, define

$$
M_{g}(x, y, t)=\eta(t)^{d(x, y)},
$$

for all $x, y \in X$, where $d(x, y)$ is an ordinary metric. It is easy to see that $\left(X, M_{g}, *\right)$ is a fuzzy metric space.

Definition 3 ([24]). A pair $(A, S)$ of self-mappings of a fuzzy metric space $\left(X, M_{g}, *\right)$ is said to satisfy the common limit in the range property with respect to mapping $\mathrm{S}$ (briefly, $\left(C L R_{S}\right)$ property), if there exists a sequence $x_{n}$ in $X$ such that

$$
\lim _{n \rightarrow \infty} A x_{n}=\lim _{n \rightarrow \infty} S x_{n}=S z, \text { for some } z \in X .
$$

Definition $4([22])$. Let $X$ be a nonempty set. A generalized metric (or $D^{*}$-metric) on $X$ is a function $D^{*}: X^{3} \rightarrow R^{+}$that satisfying the following conditions, for each $x, y, z, a \in X$ :

(1) $D^{*}(x, y, z) \geq 0$

(2) $D^{*}(x, y, z)=0$ if and only if $x=y=z$;

(3) $D^{*}(x, y, z)=D^{*}(p\{x, y, z\})$, where $\mathrm{p}$ is a permutation function;

(4) $D^{*}(x, y, z) \leq D^{*}(x, y, a)+D^{*}(a, z, z)$. The pair $\left(X, D^{*}\right)$ is called a generalized metric (or $D^{*}$-metric) space.

An example of such a function is:

$$
D^{*}(x, y, z)=d(x, y)+d(y, z)+d(z, x),
$$

where $d$ is a ordinary metric on $X$.

Definition $5([22])$. A 3-tuple $(X, M, *)$ is called a $M$-fuzzy metric space if $X$ is an arbitrary (non-empty) set, $*$ is a continuous t-norm, and $M$ is a fuzzy set in $X^{3} \times(0, \infty)$ satisfying the following conditions for each $x, y, z, a \in X$ and $t, s>0$,

$\left(M_{1}\right) M(x, y, z, t)>0$

$\left(M_{2}\right) M(x, y, z, t)=1, t>0$, if and only if $x=y=z$;

$\left(M_{3}\right) M(x, y, z, t)=M(p\{x, y, z\}, t)$, where $p$ is a permutation function;

$\left(M_{4}\right) M(x, y, a, t)^{*} M(a, z, z, s) \leq M(x, y, z, t+s)$;

$\left(M_{5}\right) M(x, y, z, \cdot):(0, \infty) \rightarrow[0,1]$ is continuous.

Lemma 1 ([22]). Let $\left(X, M_{g}, *\right)$ be a fuzzy metric space. If we define $M$ : $X^{3} \times(0, \infty) \rightarrow[0,1]$ by

$$
M(x, y, z, t)=M_{g}(x, y, t) * M_{g}(y, z, t) * M_{g}(z, x, t)
$$

for every $x, y, z \in X$ then $(X, M, *)$ is a $M$-fuzzy metric space. 
Example 2. Let $\left(X, M_{g}, *\right)$ be a fuzzy metric space defined by

$$
M_{g}(x, y, t)=\left(\frac{t}{t+1}\right)^{|x-y|}
$$

for each $t \in(0, \infty)$ and $\forall x, y \in X$, where $a * b=a b, \forall a, b \in[0,1]$.

If we define

$$
M(x, y, z, t)=\left(\frac{t}{t+1}\right)^{|x-y|+|y-z|+|z-x|}
$$

for each $t \in(0, \infty)$ and for all $x, y, z \in X$, then

$$
\begin{aligned}
M(x, y, z, t) & =\left(\frac{t}{t+1}\right)^{|x-y|+|y-z|+|z-x|} \\
& =\left(\frac{t}{t+1}\right)^{|x-y|} \cdot\left(\frac{t}{t+1}\right)^{|y-z|} \cdot\left(\frac{t}{t+1}\right)^{|z-x|} \\
& =M_{g}(x, y, t) * M_{g}(y, z, t) * M_{g}(z, x, t)
\end{aligned}
$$

by Lemma $2.1,(X, M, *)$ is a $M$-fuzzy metric space.

Lemma 2 ([23]). Let $(X, M, *)$ be a $M$-fuzzy metric space. For any $x, y, z \in$ $X$ and $t>0$, we have

$\left(\alpha_{1}\right) M(x, x, y, t)=M(x, y, y, t)$;

$\left(\alpha_{2}\right) M(x, y, z, t)$ is a nondecreasing with respect to $t$.

Lemma $3([23])$. Let $(X, M, *)$ be a $M$-fuzzy metric space with the condition $\lim _{t \rightarrow \infty} M(x, y, z, t)=1$, for all $x, y, z \in X$ and $t>0$. If for all $x, y, z \in X$ and for a number $k \in(0,1)$

$$
M(x, y, z, k t) \geq M(x, y, z, t),
$$

then $x=y=z$.

The concept of weakly compatible mapping was introduced by Jungck [10] in metric space as follows:

Definition 6 ([10]). Two self mappings $A$ and $S$ of a metric space $(X, d)$ are said to be weakly compatible if they commute at their coincidence points, that is

$$
A x=S x \text { implies that } A S x=S A x .
$$

Motivated by the above definition Sedghi and Shobe [22] defined the following:

Definition 7 ([22]). Let $A$ and $S$ be mappings from a $M$-fuzzy metric space $(X, M, *)$ into itself. Then the mappings are said to be weakly compatible if they commute at their coincidence points, that is

$$
A x=S x \text { implies that } A S x=S A x .
$$


Definition 8 ([22]). Let $A$ and $S$ be two self-mappings of a $M$-fuzzy metric space $(X, M, *)$. We say that $A$ and $S$ satisfy the property (E), if there exists a sequence $x_{n}$ such that

$$
\lim _{n \rightarrow \infty} M\left(A x_{n}, u, u, t\right)=\lim _{n \rightarrow \infty} M\left(S x_{n}, u, u, t\right)=1,
$$

for some $u \in X$ and $\forall t>0$.

Definition 9 ([22]). Let $\Theta$ denotes a family of mappings such that each $\theta \in \Theta, \theta:[0,1]^{12} \rightarrow[0,1]$, and $\theta$ is continuous and increasing in each co-ordinate variable. Also $\theta(s, s, \ldots, s)>s$, for every $s \in[0,1)$.

Sedghi and Shobe $([22])$ proved the following result:

Theorem 1. Let $A, B, S$ and $T$ be self-mappings of a $M$-fuzzy metric space $(X, M, *)$ satisfying:

(i) $A(X) \subseteq T(X), B(X) \subseteq S(X)$ and $T(X)$ or $S(X)$ is a complete $M$-fuzzy metric subspace of $X$;

(ii) the pair $(A, S)$ and $(B, T)$ are weakly compatible and $(A, S)$ or $(B, T)$ satisfy the property $(E)$;

(iii) $M(A x, B y, B z, t) \geq \theta\{$

$$
\begin{aligned}
& M(S x, T y, T z, k t), M(S x, B y, T z, k t) \\
& M(S x, T y, B z, k t), M(S x, B y, B y, k t), \\
& M(T y, B y, B z, k t), M(T y, T y, B z, k t) \\
& M(T y, B y, B y, k t), M(T y, B z, B z, k t), \\
& M(B y, T y, T z, k t), M(B y, B y, T z, k t) \\
& M(B y, T z, T z, k t), M(T z, B z, B z, k t)\} .
\end{aligned}
$$

Then $A, B, S$ and $T$ have a unique common fixed point in $X$.

We use following in our results:

Definition 10 ([20]). Let $\Psi$ be the set of all increasing and continuous functions $\psi:(0,1] \rightarrow(0,1]$, such that $\psi(t)>t$ for every $t \in(0,1)$.

Definition 11 ([4]). Define $\Phi=\left\{\phi: R^{+} \rightarrow R^{+}\right\}$, where $R^{+}=[0,+\infty)$ and each $\phi \in \Phi$ satisfies the following conditions:

$(\phi-1) \phi$ is non-decreasing;

$(\phi-2) \phi$ is continuous;

$(\phi-3) \sum_{n=0}^{\infty} \phi^{n}(t)<+\infty$, for all $t>0$, where $\phi^{n+1}(t)=\phi^{n}(\phi(t)), n \in N$.

It is easy to prove that, if $\phi \in \Phi$, then $\phi(t)<t$ for all $t>0$.

Eventually, our main findings are:

\section{MAIN SECTION}

Definition 12 defines $C L R$ property in $M$-fuzzy metric space inspired by Definition 3. 
Definition 12. A pair $(A, S)$ of self-mappings of a $M$-fuzzy metric space $(X, M, *)$ is said to satisfy the common limit in the range property with respect to mapping $S$ (briefly, $\left(C L R_{S}\right)$ property), if there exists a sequence $x_{n}$ in $X$ such that

$$
\lim _{n \rightarrow \infty} A x_{n}=\lim _{n \rightarrow \infty} S x_{n}=S u,
$$

i.e.,

$$
\lim _{n \rightarrow \infty} M\left(A x_{n}, S u, S u, t\right)=\lim _{n \rightarrow \infty} M\left(S x_{n}, S u, S u, t\right)=1
$$

for some $u \in X$ and $\forall t>0$.

Following theorem is improved result of [22].

Theorem 2. Let $A, B, S$ and $T$ be self-mappings of a $M$-fuzzy metric space $(X, M, *)$ satisfying:

(2.1) the pair $(A, S)$ and $(B, T)$ are weakly compatible and enjoy $\left(C L R_{S}\right)$ and $\left(C L R_{T}\right)$ properties, respectively;

(2.2) for all $x, y, z \in X$ and $t>0, k>1$

$$
\begin{aligned}
M(A x, B y, B z, t) \geq \theta\{ & M(S x, T y, T z, k t), M(S x, B y, T z, k t), \\
& M(S x, T y, B z, k t), M(S x, B y, B y, k t), \\
& M(T y, B y, B z, k t), M(T y, T y, B z, k t), \\
& M(T y, B y, B y, k t), M(T y, B z, B z, k t), \\
& M(B y, T y, T z, k t), M(B y, B y, T z, k t), \\
& M(B y, T z, T z, k t), M(T z, B z, B z, k t)\},
\end{aligned}
$$

where $\theta \in \Theta$, and $\theta(1,1, \ldots, 1)=1$.

Then $A, B, S$ and $T$ have a unique common fixed point in $X$.

Proof. Since the pair $(A, S)$ and $(B, T)$ enjoy $\left(C L R_{S}\right)$ and $\left(C L R_{T}\right)$ properties, respectively, therefore there exist sequences $x_{n}$ and $y_{n}$ in $X$ such that

$$
\lim _{n \rightarrow \infty} A x_{n}=\lim _{n \rightarrow \infty} S x_{n}=S x,
$$

and

$$
\lim _{n \rightarrow \infty} B y_{n}=\lim _{n \rightarrow \infty} T y_{n}=T y,
$$

for some $x, y \in X$.

We show that $S x=T y$. Suppose that $S x \neq T y$. Condition (2.2) implies that 


$$
\begin{aligned}
& M\left(A x_{n}, B y_{n}, B y_{n+1}, t\right) \geq \\
& \theta\left\{M\left(S x_{n}, T y_{n}, T y_{n+1}, k t\right), M\left(S x_{n}, B y_{n}, T y_{n+1}, k t\right),\right. \\
& M\left(S x_{n}, T y_{n}, B y_{n+1}, k t\right), M\left(S x_{n}, B y_{n}, B y_{n}, k t\right), \\
& M\left(T y_{n}, B y_{n}, B y_{n+1}, k t\right), M\left(T y_{n}, T y_{n}, B y_{n+1}, k t\right) \text {, } \\
& M\left(T y_{n}, B y_{n}, B y_{n}, k t\right), M\left(T y_{n}, B y_{n+1}, B y_{n+1}, k t\right) \text {, } \\
& M\left(B y_{n}, T y_{n}, T y_{n+1}, k t\right), M\left(B y_{n}, B y_{n}, T y_{n+1}, k t\right) \text {, } \\
& \left.M\left(B y_{n}, T y_{n+1}, T y_{n+1}, k t\right), M\left(T y_{n+1}, B y_{n+1}, B y_{n+1}, k t\right)\right\} \text {. }
\end{aligned}
$$

Take the limit as $n \rightarrow+\infty$ we get,

$$
\begin{aligned}
& M(S x, T y, T y, t) \geq \theta\{(S x, T y, T y, k t), M(S x, T y, T y, k t), \\
& M(S x, T y, T y, k t), M(S x, T y, T y, k t), \\
&1,1,1,1,1,1,1,1\}, \\
& M(S x, T y, T y, t)>M(S x, T y, T y, k t),
\end{aligned}
$$

a contradiction of $\left(\alpha_{2}\right)$. Thus, $S x=T y$.

To show that $A x=S x$. Suppose that $A x \neq S x$. Condition (2.2) implies that

$$
\begin{aligned}
& M\left(A x, B y_{n}, B y_{n+1}, t\right) \geq \\
& \theta\{ M\left(S x, T y_{n}, T y_{n+1}, k t\right), M\left(S x, B y_{n}, T y_{n+1}, k t\right), \\
& M\left(S x, T y_{n}, B y_{n+1}, k t\right), M\left(S x, B y_{n}, B y_{n}, k t\right), \\
& M\left(T y_{n}, B y_{n}, B y_{n+1}, k t\right), M\left(T y_{n}, T y_{n}, B y_{n+1}, k t\right), \\
& M\left(T y_{n}, B y_{n}, B y_{n}, k t\right), M\left(T y_{n}, B y_{n+1}, B y_{n+1}, k t\right), \\
& M\left(B y_{n}, T y_{n}, T y_{n+1}, k t\right), M\left(B y_{n}, B y_{n}, T y_{n+1}, k t\right), \\
&\left.M\left(B y_{n}, T y_{n+1}, T y_{n+1}, k t\right), M\left(T y_{n+1}, B y_{n+1}, B y_{n+1}, k t\right)\right\} .
\end{aligned}
$$

Take the limit as $n \rightarrow+\infty$, we get

$$
M(A x, S x, S x, t) \geq \theta\{1,1,1,1,1,1,1,1,1,1,1,1\}=1 .
$$

Hence, $A x=S x$. Similarly, using $\left(\alpha_{1}\right)$, we can show that $T y=B y$.

Thus, $A x=S x=T y=B y$.

Since, the pairs $(A, S)$ and $(B, T)$ are weakly compatible therefore

$$
A x=S x \text { implies that } A S x=S A x,
$$

and

$$
T y=B y \text { implies that } B T y=T B y \text {. }
$$

Thus, we have

$$
A A x=A S x=S A x=S S x
$$


and

$$
B B y=B A x=B T y=T B y=T A x=T T y .
$$

To show that $A A x=A x$. Suppose that $A A x \neq A x$. Condition (2.2) implies that

$$
\begin{aligned}
M\left(A A x, B y, B y_{n}, t\right) \geq \theta\{ & M\left(S A x, T y, T y_{n}, k t\right), M\left(S A x, B y, T y_{n}, k t\right), \\
& M\left(S A x, T y, B y_{n}, k t\right), M(S A x, B y, B y, k t), \\
& 7 M\left(T y, B y, B y_{n}, k t\right), M\left(T y, T y, B y_{n}, k t\right), \\
& M(T y, B y, B y, k t), M\left(T y, B y_{n}, B y_{n}, k t\right), \\
& M\left(B y, T y, T y_{n}, k t\right), M\left(B y, B y, T y_{n}, k t\right), \\
& \left.M\left(B y, T y_{n}, T y_{n}, k t\right), M\left(T y_{n}, B y_{n}, B y_{n}, k t\right)\right\} .
\end{aligned}
$$

Take the limit as $n \rightarrow+\infty$, we get

$$
\begin{aligned}
M(A A x, A x, A x, t) \geq \theta\{ & M(A A x, A x, A x, k t), M(A A x, A x, A x, k t), \\
& M(A A x, A x, A x, k t), M(A A x, A x, A x, k t), \\
& 1,1,1,1,1,1,1,1\}, \\
M(A A x, A x, A x, t)> & M(A A x, A x, A x, k t),
\end{aligned}
$$

a contradiction of $\left(\alpha_{2}\right)$. Thus, $A A x=A x$. Similarly, using $\left(\alpha_{1}\right)$, we can show that $B B y=B y$.

Now we have,

$$
S A x=A A x=A x=B y=B B y=B A x=T A x .
$$

Hence, $A A x=S A x=B A x=T A x=A x$, i.e. $A x$ is a common fixed point of $A, B, S$ and $T$. The uniqueness follows from (2.2).

Remark 1. Our Theorem 3.1 improves result of [22] in the following sense: Containment of ranges and completeness of the subspace have been completely removed and Property (E) is replaced by $C L R_{S}$ property.

Theorem 3. Let $A$ and $S$ be self mappings of a M-fuzzy metric space $(X, M, *)$ satisfying the following conditions:

(3.1) the pair $(A, S)$ is weakly compatible and enjoys the $C L R_{S}$ property;

(3.2) there exists a constant $k \in(0,1)$ and for all $x, y \in X, t>0$,

$$
M(A x, A y, A y, k t) \geq \psi(M(S x, S y, S y, t)),
$$

where $\psi \in \Psi$ with $\psi(1)=1$.

Then $A$ and $S$ have a unique common fixed point in $X$.

Proof. Since the pair $(A, S)$ enjoys the $C L R_{S}$ property, there exists a sequence $x_{n}$ in $X$ such that

$$
\lim _{n \rightarrow \infty} A x_{n}=\lim _{n \rightarrow \infty} S x_{n}=S u, \text { for some } u \in X .
$$


We show that $A u=S u$. Suppose that $A u \neq S u$. Using (3.2) we get,

$$
M\left(A x_{n}, A u, A u, k t\right) \geq \psi\left(M\left(S x_{n}, S u, S u, t\right)\right) .
$$

Take the limit as $n \rightarrow+\infty$ we get,

$$
\begin{gathered}
M(S u, A u, A u, k t) \geq \psi(M(S u, S u, S u, t))=\psi(1)=1 ; \\
M(S u, A u, A u, t) \geq 1 .
\end{gathered}
$$

Hence, $A u=S u$, i.e. $u$ is a coincidence point of $A$ and $S$.

Since, the pair $(A, S)$ is weakly compatible therefore

$$
A u=S u \text { implies that } A S u=S A u,
$$

and thus

$$
A A u=A S u=S A u=S S u .
$$

Next we assert that $A A u=A u$. Suppose that $A A u \neq A u$. Using (3.2), we get

$$
\begin{aligned}
M(A A u, A u, A u, k t) & \geq \psi(M(S A u, S u, S u, t)) \\
& =\psi(M(A A u, A u, A u, t)), \\
& >M(A A u, A u, A u, t),
\end{aligned}
$$

by Lemma 3 , we have, $A A u=A u$.

Hence, $A u=S A u=A u$, i.e. $A u$ is a common fixed point of $A$ and $S$ in $X$. The uniqueness follows from (3.2).

Example 3. Let $X=[0,1]$ and $d$ is a ordinary metric on $X$. Define $a * b=$ $a b$, for all $a, b \in[0,1]$,

$$
D^{*}(x, y, z)=d(x, y)+d(y, z)+d(z, x),
$$

and

$$
M(x, y, z, t)=\left(\frac{t}{t+1}\right)^{D^{*}(x, y, z)}
$$

for all $x, y, z \in X, t>0$. Then $(X, M, *)$ is $M$-fuzzy metric space. Define $A, S: X \rightarrow X$ as follows:

$$
A x=0 \text { and } x=x .
$$

Clearly, the pair $(A, S)$ enjoys the $C L R_{S}$ property for the sequence $x_{n}=$ $\frac{1}{n} \in X$, since

$$
\lim _{n \rightarrow \infty} A x_{n}=0(=S(0))=\lim _{n \rightarrow \infty} x_{n} .
$$

Also, the pair $(A, S)$ is weakly compatible, since, for $x \in X, A(x)=S(x)$ implies that $A S(x)=S A(x)$. One can easily verify that $A$ and $S$ satisfy condition (3.2), with $\psi:(0,1] \rightarrow(0,1]$ defined by $\psi(t)=t^{1 / 2}$ and $\psi(1)=1$. Thus $A$ and $S$ satisfy all the conditions of the Theorem 3 and have a common fixed point $0 \in X$. 
Theorem 4. Let $(X, M, *)$ be a $M$-fuzzy metric space where $*$ be a continuous t-norm. Let $A, S: X \rightarrow X$ be self mappings satisfying the following conditions:

(4.1) the pair $(A, S)$ is weakly compatible and enjoys the $C L R_{S}$ property;

(4.2) if there exists $\phi \in \Phi$ such that

$$
M(A x, A y, A y, \phi(t)) \geq \psi(M(S x, S y, S y, t)),
$$

for all $x, y \in X, t>0$, where $\psi \in \Psi$ with $\psi(1)=1$.

Then $A$ and $S$ have a unique common fixed point in $X$.

Proof. Since the pair $(A, S)$ enjoys the $C L R_{S}$ property, there exists a sequence $x_{n}$ in $X$ such that

$$
\lim _{n \rightarrow \infty} A x_{n}=\lim _{n \rightarrow \infty} S x_{n}=S u, \text { where } u \in X .
$$

Since, $\phi \in \Phi$ then $\phi(t)<t$ and using $\left(\alpha_{2}\right)$, we have,

$$
M\left(A x_{n}, A u, A u, t\right) \geq M\left(A x_{n}, A u, A u, \phi(t)\right) \geq \psi\left(M\left(S x_{n}, S u, S u, t\right)\right) .
$$

Take the limit as $n \rightarrow+\infty$ we get,

$$
\begin{gathered}
M(S u, A u, A u, t) \geq \psi(M(S u, S u, S u, t))=\psi(1)=1, \\
M(S u, A u, A u, t) \geq 1 .
\end{gathered}
$$

Hence, $A u=S u$, i.e. $u$ is a coincidence point of $A$ and $S$.

Since, the pair $(A, S)$ is weakly compatible therefore

$$
A u=S u \text { implies that } A S u=S A u,
$$

and thus

$$
A A u=A S u=S A u=S S u .
$$

Next we assert that $A A u=A u$. Suppose that $A A u \neq A u$. Using (4.2), we get

$$
\begin{aligned}
M(A A u, A u, A u, \phi(t)) & \geq \psi(M(S A u, S u, S u, t)) \\
& =\psi(M(A A u, A u, A u, t)), \\
& >M(A A u, A u, A u, t),
\end{aligned}
$$

a contradiction of $\left(\alpha_{2}\right)$ because $t>\phi(t)$. Thus, $A A u=A u$.

Hence, $A A u=S A u=A u$, i.e. $A u$ is a common fixed point of $A$ and $S$ in $X$. The uniqueness follows from (4.2).

Example 4. Let $X=[0,1]$ and $d$ is a ordinary metric on $X$. Define $a * b=$ $a b$, for all $a, b \in[0,1]$,

$$
D^{*}(x, y, z)=d(x, y)+d(y, z)+d(z, x),
$$

and

$$
M(x, y, z, t)=\left(\frac{t}{t+1}\right)^{D^{*}(x, y, z)}
$$


for all $x, y, z \in X, t>0$. Then $(X, M, *)$ is $M$-fuzzy metric space. Define $A, S: X \rightarrow X$ as follows:

$$
A x=\frac{x}{4} \text { and } S x=x .
$$

Clearly, the pair $(A, S)$ enjoys the $C L R_{S}$ property for the sequence $x_{n}=$ $\frac{1}{n} \in X$, since

$$
\lim _{n \rightarrow \infty} A x_{n}=0(=S(0))=\lim _{n \rightarrow \infty} S x_{n} .
$$

Also, the pair $(A, S)$ is weakly compatible, since, $A(x)=S(x)$ implies that $A S(x)=S A(x)$. One can easily verify that $A$ and $S$ satisfy condition (4.2), with $\phi(t)=\frac{t}{2}$ and $\psi:(0,1] \rightarrow(0,1]$ defined by $\psi(t)=t^{1 / 2}$. Thus $A$ and $S$ satisfy all the conditions of the Theorem 3.3 and have a common fixed point $0 \in X$.

\section{Application to integral EQUAtions}

Here, we study the existence and uniqueness of the solution of a Volterra integral equations of the second kind, using our results. Inspired by [12], we consider the integral equation

$$
x(r)=g(r)+\int_{0}^{r} K(r, s, x(s)) d s,
$$

for all $r \in[0, I]$, where $I>0$.

In view of [12], $X=C([0, I], R)$ be the space of all continuous functions defined on $[0, I]$ endowed with the supremum norm

$$
\|x\|=\sup _{r \in[0, I]}|x(r)|, \quad x \in X,
$$

and the induced metric

$$
d(x, y)=\sup _{r \in[0, I]}|x(r)-y(r)|, \quad x, y \in X .
$$

Here, we study the existence of the solution of integral equation (1) in $X$, using our results.

We define $D^{*}: X^{3} \rightarrow R^{+}$by

$$
D^{*}(x, y, z)=d(x, y)+d(y, z)+d(z, x) .
$$

Then $\left(X, D^{*}\right)$ is a $D^{*}$-metric space.

Denote $a * b=a b, \forall a, b \in[0,1]$, and for each $t \in(0, \infty)$, we define,

$$
M(x, y, z, t)=\eta(t)^{D^{*}(x, y, z)},
$$

for all $x, y, z \in X$, where $\eta(t)=\frac{t}{t+1}$. Then $(X, M, *)$ is a $M$-fuzzy metric space induced by $D^{*}$-metric as shown in Example 2.

Now, we prove the existence of the solution of (1). 
Theorem 5. Let $T: X \rightarrow X$ be the integral operator given by

$$
T(x(r))=g(r)+\int_{0}^{r} K(r, s, x(s)) d s, \quad g \in X
$$

where $K \in C([0, I] \times[0, I] \times R, R)$ satisfies the following conditions:

(5.1) there exists $m:[0,1]^{2} \rightarrow R^{+}$such that for each $r, s \in[0, I]$ and $x, y \in X$,

$$
|K(r, s, x(s))-K(r, s, y(s))| \leq m(r, s)|x(s)-y(s)|,
$$

(5.2) for some $0 \leq \lambda<1$,

$$
\sup _{r \in[0, I]} \int_{0}^{r} m(r, s) d s \leq \lambda,
$$

where $\int_{0}^{t} m(r, s) d s$ is bounded on $[0, I]$.

Then the integral equation (1) has a unique solution $x^{\prime} \in X$.

Proof. Let $X=C([0, I], R)$. We can verify that $(X, M, *)$ is a $M$-fuzzy metric space with $a * b=a b$, for all $a, b \in[0,1]$, and $M(x, y, z, t)$ is as given in (2). Obviously, $\eta(t)$ is an increasing function and $\eta(t) \in(0,1)$.

For all $x, y \in X$, we have,

$$
\begin{aligned}
D^{*}(T x, T y, T y) & =2 \sup _{r \in[0, I]}|T(x(r))-T(y(r))| \\
& \leq 2 \sup _{r \in[0, I]} \int_{0}^{r}|K(r, s, x(s))-K(r, s, y(s))| d s \\
& \leq 2 \sup _{r \in[0, I]} \int_{0}^{r} m(r, s)|x(s)-y(s)| d s \\
& \leq D^{*}(x, y, y) \sup _{r \in[0, I]} \int_{0}^{r} m(r, s) d s .
\end{aligned}
$$

By using (5.2), there is $0 \leq \lambda<1$, such that

$$
\sup _{r \in[0, I]} \int_{0}^{r} m(r, s) d s \leq \lambda .
$$

Thus we have,

$$
D^{*}(T x, T y, T y) \leq \lambda D^{*}(x, y, y) .
$$

For all $t \geq 0$, we have

$$
\left(\frac{\frac{t}{2}}{\frac{t}{2}+1}\right) \geq\left(\frac{t}{t+1}\right)^{2}
$$

Since, $\eta(t) \in(0,1)$ and in view of $(2)$, we have

$$
M\left(T x, T y, T y, \frac{t}{2}\right)=\left(\frac{\frac{t}{2}}{\frac{t}{2}+1}\right)^{D^{*}(T x, T y, T y)}
$$




$$
\begin{aligned}
& \geq\left[\left(\frac{t}{t+1}\right)^{2}\right]^{D^{*}(T x, T y, T y)} \\
& \geq\left(\frac{t}{t+1}\right)^{2 \lambda D^{*}(x, y, y)} \\
& =\left[\left(\frac{t}{t+1}\right)^{D^{*}(x, y, y)}\right]^{2 \lambda} \\
& =(M(x, y, y, t))^{2 \lambda} \\
& =\psi(M(x, y, y, t)),
\end{aligned}
$$

where $\psi(n)=\left(n^{\lambda}\right)^{2}$, for some $0 \leq \lambda<1$ and for every $n \in(0,1]$, which is increasing and continuous function.

Therefore the condition (4.2) of Theorem 4 with $f=T, g=I$ (Identity mapping) and $\phi(t)=\frac{t}{2}$ is satisfied. This shows that all the conditions of Theorem 3.3 are satisfied. This proves that $T$ has a unique fixed point $x^{\prime} \in X=C([0, I], R)$, which is a solution of the integral equation (1).

Example 5. Find the solution of the integral equation

$$
T(x(r))=1+r+\int_{0}^{r}(r-s) x(s) d s,
$$

for all $r \in[0, I]$, where $I=1>0$.

Solution. Using integral equation of Theorem 5 and equation (3), we have

$$
g(r)=1+r \in C([0,1], R) \text { and } K(r, s, x(s))=(r-s) x(s),
$$

then there exists $m:[0,1]^{2} \rightarrow R^{+}$such that for each $r, s \in[0,1]$ and $x, y \in X$,

$$
|K(r, s, x(s))-K(r, s, y(s))|=m(r, s)|x(s)-y(s)|,
$$

where $m(r, s)=|r-s|$. Thus (5.1) holds.

One can easily check that for some $\lambda \in[0,1)$,

$$
\sup _{r \in[0,1]} \int_{0}^{r}(r-s) d s \leq \lambda \text {, }
$$

(i.e. $\left.\frac{r^{2}}{2} \leq \lambda\right)$ holds for $r \in[0,1]$, where $\int_{0}^{r}(r-s) d s$ is bounded on $[0,1]$. Thus (5.2) holds.

Hence, $T$ satisfies all the conditions of the Theorem 5 then there must exists a solution of the integral equation (3), which is also a unique fixed point of $T$.

To find the fixed point of $T$ : $T$ can have a fixed point if $T(x(r))=x(r)$, then using (3) we have an integral equation

$$
x(r)=1+r+\int_{0}^{r}(r-s) x(s) d s .
$$


We use the Laplace Transform method to solve integral equation (4). We can rewrite (4) as

$$
x(r)=(1+r)+r \star x(r),
$$

where $r \star x(r)$ is the convolution product of $r$ and $x(r)$.

Taking Laplace transform on both side to equation (5), we have

$$
\mathcal{L}[x(r)]=\frac{1}{p}+\frac{1}{p^{2}}+\mathcal{L}[r \star x(r)]=\frac{p+1}{p^{2}}+\frac{1}{p^{2}} \mathcal{L}[x(r)],
$$

i.e.,

and thus

$$
\mathcal{L}[x(r)]=\frac{1}{p-1}
$$

$$
x(r)=\mathcal{L}^{-1}\left[\frac{1}{p-1}\right]=e^{r} .
$$

Hence $x(r)=e^{r} \in C([0,1], R)$ is a solution of the integral equation, which is also a unique fixed point of $T$.

Example 6. Find the solution of the integral equation

$$
T(x(r))=1+\int_{0}^{r} \alpha x(s) d s,
$$

for all $r \in[0, I]$, where $I=\frac{1}{2}>0$ and $0<\alpha<1$.

Solution. Using integral equation of Theorem 5 and equation (6), we have $g(r)=1 \in C\left(\left[0, \frac{1}{2}\right], R\right)$ and $K(r, s, x(s))=\alpha x(s)$, then there exists $m$ : $[0,1]^{2} \rightarrow R^{+}$such that for each $r, s \in\left[0, \frac{1}{2}\right], x, y \in X$ and $\alpha<1$,

$$
|K(r, s, x(s))-K(r, s, y(s))|=\alpha|x(s)-y(s)|<m(r, s)|x(s)-y(s)|,
$$

where $m(r, s)=1$. Thus (5.1) holds.

One can easily check that

$$
\sup _{r \in\left[0, \frac{1}{2}\right]} \int_{0}^{r} d s \leq \lambda,
$$

(i.e. $r \leq \lambda$ ) holds for $r \in\left[0, \frac{1}{2}\right]$ and some $\lambda \in[0,1)$, where $\int_{0}^{r} d s$ is bounded on $[0,1]$. Thus (5.2) holds.

Hence, $T$ satisfies all the conditions of the Theorem 5 , then there exists a solution of the integral equation (6), which is also a fixed point of $T$.

To find the fixed point of $T$ : $T$ can have a fixed point if $T(x(r))=x(r)$, then using (6) we have an integral equation

$$
x(r)=1+\int_{0}^{r} \alpha x(s) d s .
$$

Using Laplace Transform method, we can rewrite (7) as

$$
x(r)=1+\alpha[1 \star x(r)],
$$


where $1 \star x(r)$ is the convolution product of 1 and $x(r)$. Taking Laplace transform on both side to equation (8), we have

$$
\mathcal{L}[x(r)]=\frac{1}{p}+\alpha \mathcal{L}[1 \star x(r)]=\frac{1}{p}+\frac{\alpha}{p} \mathcal{L}[x(r)],
$$

i.e.,

$$
\mathcal{L}[x(r)]=\frac{1}{p-\alpha}
$$

and thus

$$
x(r)=\mathcal{L}^{-1}\left[\frac{1}{p-\alpha}\right]=e^{\alpha r},
$$

where $0<\alpha<1$.

Hence $x(r)=e^{\alpha r} \in C([0,1], R)$ is a solution of the integral equation, which is also a unique fixed point of $T$.

\section{Conclusion}

We have defined common limit in the range property in $M$-fuzzy metric space and also established some common fixed point theorem under the same property in the aforesaid space, which is an improved result of [22]. Further, we have obtained fixed point theorem using $\phi$-contractive condition in such spaces with the illustration of an example. We have studied an application of our theorem to solve Volterra integral equations of the second kind with instances.

\section{ACKNOWLEDGEMENTS}

The authors would like to express his sincere thanks to the editor and the anonymous referee for their valuable comments and useful suggestions in improving the manuscript.

\section{REFERENCES}

[1] M. Aamri and D. El Moutawakil, Some new common fixed point theorems under strict contractive conditions, Journal of Mathematical Analysis and Applications, 270 (1) (2002), 181-188.

[2] J. Chu and P. J. Torres, Application of Schauder's fixed point theorem to singular differential equations, Bulletin of the London Mathematical Society, 39 (2007), 653660 .

[3] B. Deshpande and A. Handa, Application of coupled fixed point technique in solving integral equations on modified intuitionistic fuzzy metric spaces, Advances in Fuzzy Systems, (2014), Article ID: 348069, 11 pages.

[4] B. Deshpande, S. Sharma and A. Handa, Common coupled fixed point theorems for nonlinear contractive condition on intuitionistic fuzzy metric spaces with application to integral equations, Journal of the Korean Society of Mathematical Education. Series B. The Pure and Applied Mathematics, 20 (3) (2013), 159-180. 
[5] B. C. Dhage, Generalized metric space and mapping with fixed point, Bulletin of the Calcutta Mathematical Society, 84 (4) (1992), 329-336.

[6] S. Gahler, 2-metrische Raume und ihre topologische Struktur, Mathematische Nachrichten, 26 (1963), 115-148.

[7] M. Gangopadhyay, P.Dan and M. Saha, An application of random fixed point theorem in integral equation, IOSR Journal of Mathematics, 9 (4)(2014), 57-61.

[8] A. George and P. Veeramani, On some results in fuzzy metric spaces, Fuzzy Sets and Systems, 64 (3) (1994), 395-399.

[9] M. Gugnani, M. Aggarwal and R. Chugh, Common fixed point results in G-metric spaces and applications, International Journal of Computer Applications, 43 (11) (2012), 38-42.

[10] G. Jungck, Common fixed points for noncontinuous nonself maps on nonmetric spaces, Far Eastern Journal of Mathematics and Science, 4 (1996), 199-215.

[11] N. Mani, Generalized $C_{\beta}^{\psi}$-rational contraction and fixed point theorem with application to second order differential equation, Mathematica Moravica, 22 (1) (2018), 43-54.

[12] U. Mishra, C. Vetro and P. Kumam, On modified $\alpha$ - $\phi$-fuzzy contractive mappings and an application to integral equations, Journal of Inequalities and Applications, 2016 (2016), Article ID: 67, 15 pages.

[13] Z. Mustafa and B. Sims, Some Remarks Concerning D-Metric Spaces, Proceedings of the International Conferences on Fixed Point Theory and Applications, Valencia (Spain), July 2003, 189-198.

[14] R. Muthuraj and R. Pandiselvi, Common fixed point theorems for weakly compatible of four mappings in generalized fuzzy metric spaces, International Journal of Mathematics and its Applications, 3 (1) (2015), 39-47.

[15] S. V. R. Naidu, K. P. R. Rao, and N. Srinivasa Rao, On the topology of D-metric spaces and generation of D-metric spaces from metric spaces, International Journal of Mathematics and Mathematical Sciences, 2004 (51) (2004), 2719-2740.

[16] V. Popa, A general fixed point theorem for two hybrid pairs of mappings satisfying a mixed implicit relation and applications, Mathematica Moravica, 21 (2) (2017), 103-114.

[17] K.P.R. Rao, G.N.V. Kishore, K.V. Siva Parvathi, A quadruple fixed point theorem for contractive type condition by using ICS mapping and application to integral equation, Mathematica Moravica, 18 (2) (2014), 21-34.

[18] R. Saadati, Existence and uniqueness of solutions for a class of integral equations by common fixed point theorems in IFMT-spaces, Journal of Inequalities and Applications, 2016 (2016), Article ID: 205, 8 pages.

[19] B. Schweizer and A. Sklar, Statistical metric spaces, Pacific Journal of Mathematics, 10 (1) (1960), 313-334.

[20] S.Sedghi, N. Shobe and A. Aliouche, A common fixed point theorem for weakly compatible mappings in fuzzy metric space, General Mathematics, 18 (3) (2010), 3-12.

[21] S. Sedghi, N. Shobe and H. Zhou, A Common fixed point theorem in $D^{*}$-metric spaces, Fixed Point Theory and Applications, 2007 (2007), Article ID: 27906, 1-13. 
[22] S. Sedghi and N. Shobe, Fixed point theorems in M-fuzzy metric spaces with property (E), Advances in Fuzzy Mathematics, 1(1) (2006), 55-65.

[23] D. Singh, M. Sharma and R. Sharma, Fixed point theorems in M-fuzzy metric spaces, Annals of Fuzzy Mathematics and Informatics, 5 (1) (2013), 147-155.

[24] W. Sintunavarat and P. Kumam, Common fixed point theorems for a pair of weakly compatible mappings in fuzzy metric spaces, Journal of Applied Mathematics, 2011 (2011), Article ID: 637958, 14 pages.

[25] S. Sharma and B. Deshpandey, Fixed point theorem and its application to best approximation theory, Bulletin of the Calcutta Mathematical Society, 93 (2) (2001), 155-166.

Ved Prakash BhardWaJ

Department of Mathematics

Govt. Arts, Commerce And

Science College

SUKHTAWA

Hoshangabad, M.P., 461001

INDIA

E-mail address: ved_bhar2@rediffmail.com

KaMAL WADHWA

Department of Mathematics

Govt. P.G. College, Pipariya

Hoshangabad, M.P., 461001

INDIA

E-mail address: wadhwakamal68@gmail.com 